\title{
Defect control at nanoscale and flux pinning enhancement in $\mathrm{MgB}_{2}$ superconductor
}

\author{
C.H. CHENG ${ }^{*}$, C. SORRELL \\ School of Materials Science and Engineering, University of New South Wale, Sydney 2052, NSW, Australia
}

\begin{abstract}
Defect control at nanoscale of $\mathrm{MgB}_{2}$ by doping various nanoparticles including Ti, $\mathrm{C}$, nano-diamond, and $\mathrm{HoB}_{4}$, and their roles played to enhance flux pinning force in $\mathrm{MgB}_{2}$ are compared and analyzed. These nanodopants have different chemical and physical properties, thus bring about different pinning efficiency, especially nanodopants with strong magnetic moment are particularly interesting as pinning centers in $\mathrm{MgB}_{2}$ since magnetic impurities usually have a stronger interaction with magnetic flux line than nonmagnetic impurities and may exert a stronger force to trap the flux lines when they are properly introduced into the superconducting matrix.
\end{abstract}

Key words: $\mathrm{MgB}_{2}$; flux pinning; nano-pinning centers; microstructure control

(C) 2012 JMT. All rights reserved.

\section{Introduction}

$\mathrm{L}$ arge-scale superconducting devices for power applications depend on the conductors with high critical current densities at temperatures where the costs for coolant are affordable. The discovery of superconductivity at $39 \mathrm{~K}$ in $\mathrm{MgB}_{2}$ [1] offers the possibility of wide engineering applications in a temperature range of $20-30 \mathrm{~K}$, where the conventional superconductors cannot play any roles because of low $T_{\mathrm{c}}$. However, the commercialization of $\mathrm{MgB}_{2}$-based superconducting technology depends critically on continuous improvement of the performance of $\mathrm{MgB}_{2}$ material, especially the properties in high magnetic fields, including $H_{\mathrm{c} 2}$ and $J_{\mathrm{c}}$.

Among many methods, alloying with carbon seems to be the most effective to improve the $H_{\mathrm{c} 2}$ by shorting the mean free length of electron [2]. Besides the efforts of increasing $H_{\mathrm{c} 2}$ by carbon doping, many efforts have been focused on the control of nanostructure of $\mathrm{MgB}_{2}$ in order to introduce nano-pinning-centers in the system and then increase the $J_{\mathrm{c}}$. There are a variety of types of nanoparticles used to doping the $\mathrm{MgB}_{2}$ matrix and modify its nanostructure in order to control the pinning structure of the $\mathrm{MgB}_{2}$ superconductors. Typical nanoscale doping materials used for this purpose include $\mathrm{Ti}, \mathrm{Zr}$, nanodiamond, $\mathrm{HoB}_{4}$, etc., and their effects on flux pinning behavior of $\mathrm{MgB}_{2}$ are quite striking [3-7]. However, the

Received May 10, 2012; revision accepted Jun. 6, 2012

*Corresponding author. E-mail: chengcecily@gmail.com (C.H. CHENG)

(C) 2012 JMT. All rights reserved

doi: 10.3969/j.issn.2095-087X.2012.02.001 chemical and/or physical properties of these nanodopants are very different from each other, the underpinning mechanisms are not well understood up to date, thus it is necessary to clarify them in order to optimize the nanopinning structure for a better performance.

In this paper, magnetic and nonmagnetic nanopartical doping effect has been analyzed against their flux pinning behavior for $\mathrm{MgB}_{2}$ superconductor. We identify that nanodopants with strong magnetic moment are particularly interesting as pinning centers in $\mathrm{MgB}_{2}$ since magnetic impurities usually have a stronger interaction with magnetic flux line than nonmagnetic impurities and may exert a stronger force to trap the flux lines when they are properly introduced into the superconducting matrix. This suggests that pinning sites with strong magnetic moment may play an important role to further improve the pinning behavior of $\mathrm{MgB}_{2}$ in practical applications.

\section{Experimental}

Typical procedures for the preparation of the samples are: samples with a nominal composition are prepared with solid state reaction method with starting powder materials of amorphous B (99.99\%), Mg (99.9\%), and the nano-particle dopants. The particle sizes of magnesium and boron are about 1 Micron and $200 \mathrm{~nm}$, respectively. The size of the nano dopants is about $10-50 \mathrm{~nm}$. After well ground in a glove box for $1 \mathrm{~h}$, the mixed powders were pressed into pellets of a diameter of $10 \mathrm{~mm}$, sealed in iron tubes with excess $\mathrm{Mg}$, sintered at $850{ }^{\circ} \mathrm{C}$ for $2 \mathrm{~h}$ in flowing $\mathrm{Ar}$, and finally quenched to room temperature. 
Crystalline structure was studied by powder X-ray diffraction (XRD) using an X'Pert MRD diffractometer with $\mathrm{Cu} K$-alpha radiation. Microstructure was analyzed with a scanning electron microscope (SEM) and a Philips field emission transmission electron microscope with energydispersive X-ray spectroscopy (EDX) analysis. Magnetization was measured using a $9 \mathrm{~T}$ physical property measurement system (Quantum design). The typical sample size is $0.8 \times 0.8 \times 1.0 \mathrm{~mm}^{3}$. A magnetic $J_{\mathrm{c}}$ was derived from the width of the magnetization loop $\Delta M$ based on the extended Bean model: $J_{\mathrm{c}}=20 \Delta M /[a(1-a / 3 b)]$. $H_{\text {irr }}$ was determined from emerging point of $M(T)$ curve measured in zero-field-cooling (ZFC) and field-cooling (FC) processes at various fields up to $7 \mathrm{~T}$.

\section{Results and discussion}

\subsection{Nano-diamond doping effect}

The $\mathrm{MgB}_{2}$-diamond nanocomposites with compositions of $\mathrm{MgB}_{2-x} \mathrm{C}_{x}(x=0 \%, 5 \%, 8 \%$, and $10 \%)$ were prepared. The size of nanodiamond powder is $\sim 10-20 \mathrm{~nm}$. These samples were sintered at a relatively high temperature of $800{ }^{\circ} \mathrm{C}$ for a relatively long time of $2 \mathrm{~h}$ in order to emphasize the carbon substitution effect on B. Another sample with an added $1.5 \mathrm{wt} \%$ of nanodiamond in $\mathrm{MgB}_{2}$ was also prepared but at a relatively low temperature of $730{ }^{\circ} \mathrm{C}$ for a relatively short time of $30 \mathrm{~min}$ in order to reduce the substitution effect of carbon in boron in $\mathrm{MgB}_{2}$ and emphasize the additional effect of the nanodiamond in $\mathrm{MgB}_{2}$.

As shown in Fig. 1(a), the diamond substitutional sample mainly consists of relatively large $\mathrm{MgB}_{2}$ grains ( $\sim 1 \mu \mathrm{m}$ in size) with a high density of dislocations. In some areas, discrete nano-sized particles can be observed. These dislocations mainly occur near the nanodiamond particles. This clearly shows that the unreacted carbon can introduce high density of dislocation loops in the $\mathrm{MgB}_{2}$ matrix. This phenomenon was first noted in 2003 [8-10], but its effect was not fully recognized.

As shown in Fig. 1(b), these nano-diamond doped samples exhibit significant improvements of $J_{\mathrm{c}}$, especially in high magnetic field and low temperature rejoin. This can be attributed to the substitution effect of $\mathrm{C}$ which enhances $H_{\mathrm{c} 2}$, thus consequently $J_{\mathrm{c}}$. The C substation effect is usually due to the impurity scattering effect on electrons. What we would like to point out here, the high density of dislocations induced by nanodiamond may also introduce structure-imperfection scattering effect on electrons which also enhances the $H_{\mathrm{c} 2}$ of the system. Such a dislocation enhanced $H_{\mathrm{c} 2}$ should be paid attention in $\mathrm{MgB}_{2}$ system as an alternative of improving the performance of the superconductor.
Different from the diamond substitutional sample which mainly consists of relatively large $\mathrm{MgB}_{2}$ grains $(\sim 1 \mu \mathrm{m})$ with a high density of dislocations, the diamond-added sample mainly consists of two kinds of nanoparticles: $\mathrm{MgB}_{2}$ grains with a size of $50-100 \mathrm{~nm}$ and diamond particles with a size of 10-20 nm (the results are not shown here). In fact, this diamond-added $\mathrm{MgB}_{2}$ forms a typical nano-composite material.

The nano-diamond particles are inserted into the $\mathrm{MgB}_{2}$ grains. As the $a b$-plane coherence length of $\mathrm{MgB}_{2}$ is about 6-7 nm [11], these 10 to 20 -nm-sized diamond inclusions, with a high density, are ideal flux pinning centers and are responsible for the high performance in our samples. The effect of diamond doping on the enhancement of flux pinning in $\mathrm{MgB}_{2}$ can be counterbalanced by its suppression on superconductivity, as clearly shown in the situation of $T=30 \mathrm{~K}$. This counterbalancing effect
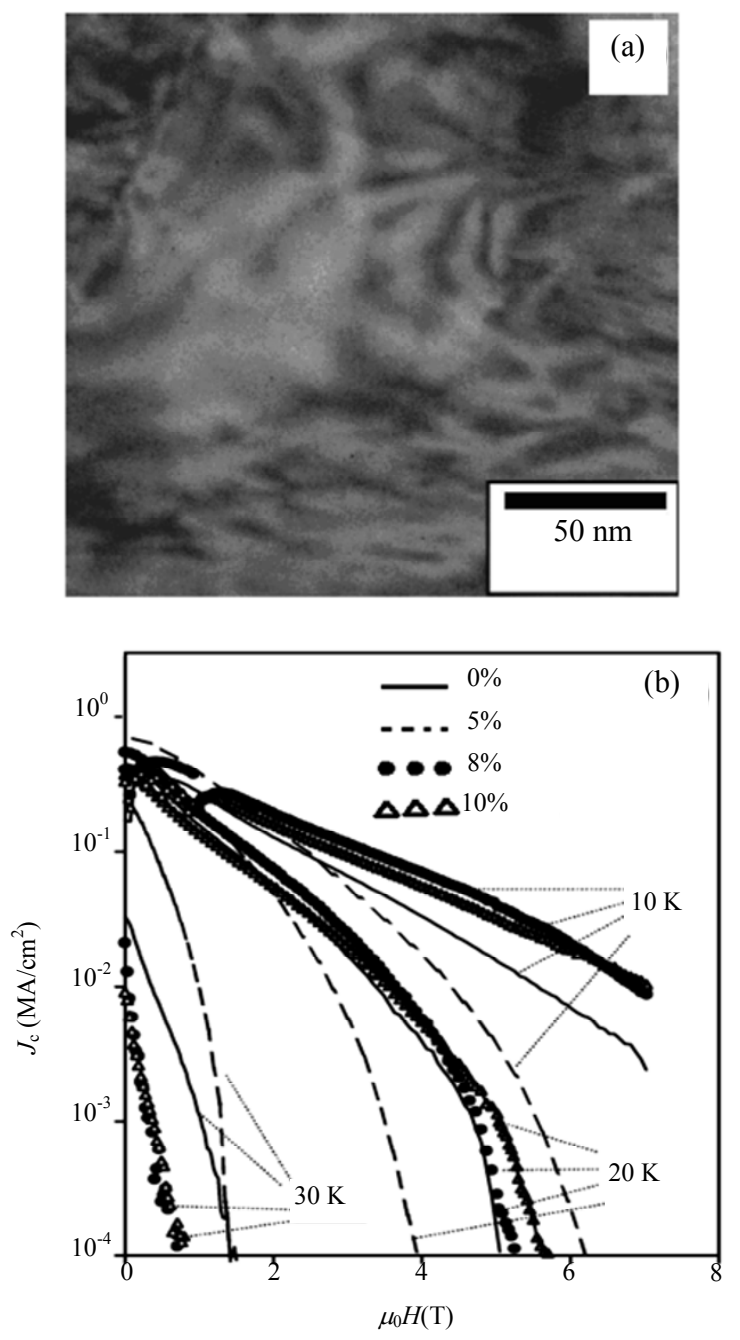

Fig. 1 (a) FEGTEM micrographs for: diamond-substituted $\mathrm{MgB}_{2}$ with $x=5 \%$. (b) Magnetic field dependence of $J_{\mathrm{c}}$ at 10 , 20 and $30 \mathrm{~K}$ for nano-diamond substituted $\mathrm{MgB}_{2}$ with $x=0 \%$ (dashed lines), 5\% (solid lines), $8 \%$ (solid circles) and 10\% (opened triangles). 
may also exist at other temperatures, even when the effect of the $J_{\mathrm{c}}$ enhancement is dominant. The further increase of $J_{\mathrm{c}}$ depends critically on reducing the $T_{\mathrm{c}}$-suppression effect in the $\mathrm{MgB}_{2}$-diamond composite. This idea is confirmed by the results obtained in the diamond-added sample, $1.5 \mathrm{wt} \% \mathrm{C}$, which has a higher $T_{\mathrm{c}}$ than other diamond-doped samples and contains more nanodiamond inclusions as revealed by the transmission electron microscopy analysis. As revealed experimentally, the diamond-added sample shows a much better $J_{\mathrm{c}}-H$ behavior than the carbon-substituted sample. Its $J_{\mathrm{c}}$ reaches $1 \times 10^{4} \mathrm{~A} / \mathrm{cm}^{2}$ at $20 \mathrm{~K}$ and $4 \mathrm{~T}$, and its $H_{\text {irr }}$ reaches $6.4 \mathrm{~T}$ at $20 \mathrm{~K}$. In fact, at all temperatures below $35 \mathrm{~K}$, the $J_{\mathrm{c}}-H$ behavior (results at $20 \mathrm{~K}$ are shown here only) and the $H_{\text {irr }}-T$ relation of the diamond-added sample are much better than those of other samples in this study.

\subsection{Carbon and Ti concurrent doping effect}

Although carbon doping can significantly increase the upper critical field of $\mathrm{MgB}_{2}$, and thus improve the $J_{\mathrm{c}}-H$ behavior at high field, however, the critical current density $J_{\mathrm{c}}$ of $\mathrm{MgB}_{2}$ bulks sintered at ambient pressure is still inferior to the conventional A15 compound superconductors and $\mathrm{Nb}$ - $\mathrm{Ti}$ due to a poor connection between grains and the lack of flux pinning centers in the materials. It is expected that the pinning behavior in carbondoped $\mathrm{MgB}_{2}$ may be further improved if its microstructure can be improved. Ti may be a good candidate for this purpose since doping $\mathrm{Ti}$ in $\mathrm{MgB}_{2}$ could highly improve the superconducting properties of $\mathrm{MgB}_{2}$ in low field by controlling the microstructure of $\mathrm{MgB}_{2}$ (see Fig. 2) [4-5]. Therefore, it is very possible to improve both $H_{\mathrm{c} 2}$ and $J_{\mathrm{c}}$ of $\mathrm{MgB}_{2}$ simultaneously by concurrently doping carbon and $\mathrm{Ti}$, thus further improving its performance, especially in the higher magnetic field regime.

By taking the advantages of concurrent doping of $\mathrm{Ti}$ and $\mathrm{C}$, the microstructure of Ti-C-doped $\mathrm{MgB}_{2}$ is further improved. The inset of Fig. 3 shows the typical magnetization hysteresis loops measured at $20 \mathrm{~K}$ for the undoped, Ti-doped, and C-Ti- doped $\mathrm{MgB}_{2}$ samples and the $J_{\mathrm{c}}(H)$ curves for the typical samples at $T=20 \mathrm{~K}$. Magnetization hysteresis loops at 10 and $5 \mathrm{~K}$ are similar to those at $20 \mathrm{~K}$. In this study, the focus is the improvement of superconducting performance at $20 \mathrm{~K}$ because, at this temperature, the conventional superconductors, such as $\mathrm{Nb}_{3} \mathrm{Sn}$ and $\mathrm{Nb}$ $\mathrm{Ti}$ alloy, can no longer be used due to a lower $T_{\mathrm{c}}$. For the undoped $\mathrm{MgB}_{2}$, magnetization hysteresis loop is relatively narrow and small, suggesting a relatively low $J_{\mathrm{c}}$ and a low irreversibility field $H_{\text {irr. }}$. After it was doped with $\mathrm{Ti}$, the loop width, $\Delta M$ is significantly enlarged at low fields. However, the loop width decreases rapidly with increasing the applied magnetic field, suggesting a degradation of the flux pinning in the magnetic field. By further doping $\mathrm{C}$ in Ti-doped $\mathrm{MgB}_{2}$, the rapid decrease of $\Delta M$ is suppressed.

As shown in the main panel of Fig. 3, the in-field critical current densities were enhanced by Ti doping, particularly in lower fields. At $20 \mathrm{~K}$, the maximum zero field value $J_{\mathrm{c}}(0)$ was achieved in $\mathrm{Mg}_{0.95} \mathrm{Ti}_{0.05} \mathrm{~B}_{2}$ sample, and a $J_{\mathrm{c}}$ value in self-field at $20 \mathrm{~K}$ was improved from $2.1 \times 10^{5} \mathrm{~A} / \mathrm{cm}^{2}$ of undoped sample to $3.1 \times 10^{5} \mathrm{~A} / \mathrm{cm}^{2}$. This result is consistent with the result of Ti-doped $\mathrm{MgB}_{2}$ bulk superconductor, where $J_{\mathrm{c}}(0)$ was improved by refining $\mathrm{MgB}_{2}$ grains and forming a strongly coupled nanoparticle structure [5].

The most striking feature shown in Fig. 3 is that the C-Ti-doped sample exhibited the combined benefits of $\mathrm{C}$ and Ti. The $J_{\mathrm{c}}$ values of this sample is lower than that of Ti-doped sample in magnetic field from 0 to $2 \mathrm{~T}$, but in higher magnetic fields $(>2 \mathrm{~T})$, its $J_{\mathrm{c}}$ is significantly improved and much higher than the $J_{\mathrm{c}}$ of Ti-doped sam-
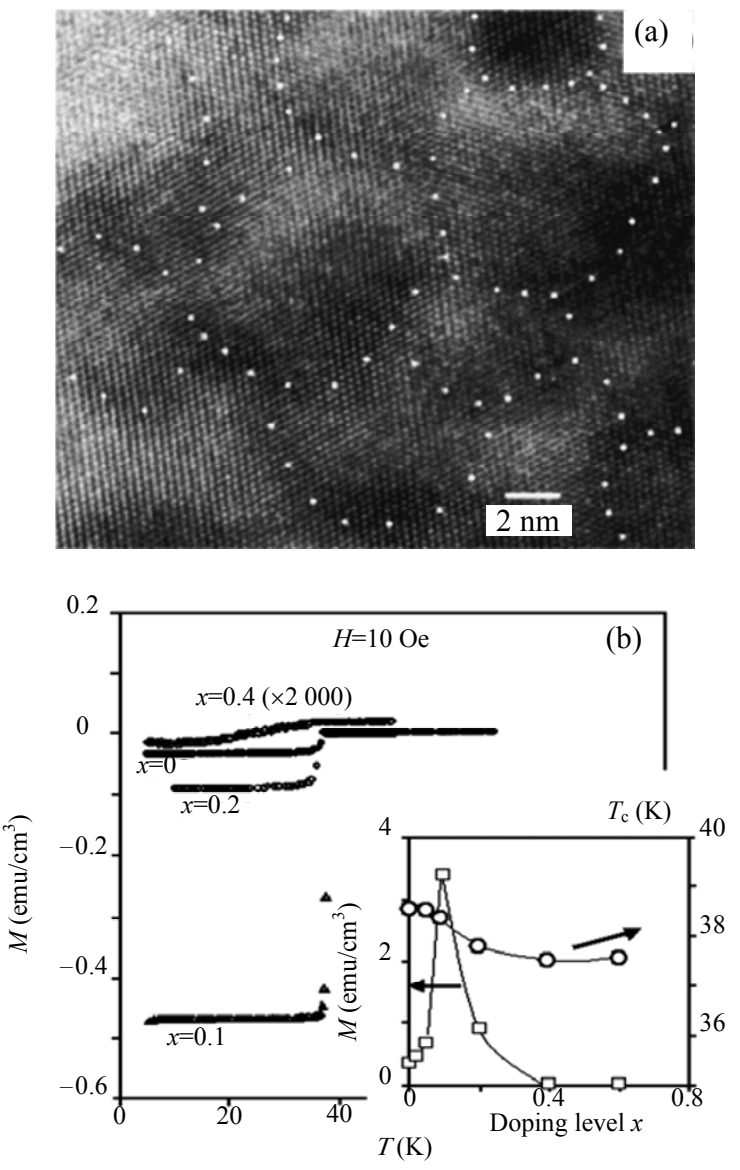

Fig. 2 HRTEM photograph for Ti-doped $\mathrm{MgB}_{2}(x=10 \%)$. A dotted network is drawn for convenience to enable one to see the nanoparticle structure. (b) Volume magnetization vs temperature curves for Ti-doped $\mathrm{MgB}_{2}$ with doping levels of $x=0$, $0.1,0.2$, and 0.4 , measured at $10 \mathrm{Oe}$ in the $\mathrm{ZFC}$ process. The data for the sample with $x=0.4$ are multiplied by a factor of 2000 . Inset: variation of critical temperature $T_{\mathrm{c}}$ and the diamagnetic signal represented by the volume magnetization at $5 \mathrm{~K}$ and $10 \mathrm{Oe}$ with doping level $x$ for Ti-doped $\mathrm{MgB}_{2}$ samples. 
ple. Accordingly, the irreversibility field is also significantly improved. What is more, this sample showed a better in-field $J_{\mathrm{c}}$ than carbon doped samples, including nanocarbon-doped $\mathrm{MgB}_{2}[12]$ and $\mathrm{CNT}$-doped $\mathrm{MgB}_{2}$ [13] in the high magnetic field region. At temperature of $20 \mathrm{~K}$, the $J_{c}$ reaches $1 \times 10^{4} \mathrm{~A} / \mathrm{cm}^{2}$ in $4 \mathrm{~T}$, and $4 \times 10^{3} \mathrm{~A} / \mathrm{cm}^{2}$ in $5 \mathrm{~T}$; and the irreversibility field determined from the closure of hysteresis loops with a criterion of $10^{2} \mathrm{~A} / \mathrm{cm}^{2}$ exceeds $6 \mathrm{~T}$. This is one of the best values for $\mathrm{MgB}_{2}$ at $20 \mathrm{~K}$.

\subsection{Nano $\mathrm{Ho}_{2} \mathrm{O}_{3}$ doping effect}

Magnetic impurities, as an easy way for magnetic flux penetration, will exert a stronger force to trap the flux lines if they can be properly introduced into the superconducting matrix. Therefore, pinning sites with strong magnetic moment is always of interest to explore the new route to enhance the pinning behavior of $\mathrm{MgB}_{2}$.

As shown in Fig. 4, SEM micrograph shows that the samples are tightly packed $\mathrm{MgB}_{2}$ nanoparticle structure with an average particle size of 50-100 nm.

As mentioned previously this type of nanostructure in $\mathrm{MgB}_{2}$ provides a good grain connection as well as the grain boundary flux pinning, sustaining a high $J_{c}$ in low and medium high field regions $(<4 \mathrm{~T})$ for $\mathrm{MgB}_{2}$. Further, TEM micrograph reveals that highly disperse nanoparticles with a size of $5-10 \mathrm{~nm}$ are inserted in the $\mathrm{MgB}_{2}$ matrix. EDX analysis reveals that these nanopar ticles contain mainly Ho and B. Combining with the XRD analyses it can be deduced that these nanoparticles are $\mathrm{HoB}_{4}$ [14-15]. This clearly confirms that doping $\mathrm{Ho}_{2} \mathrm{O}_{3}$ nanoparticles results in the formation of $\mathrm{HoB}_{4}$ nanoparticles which are weak magnetic in physical properties.

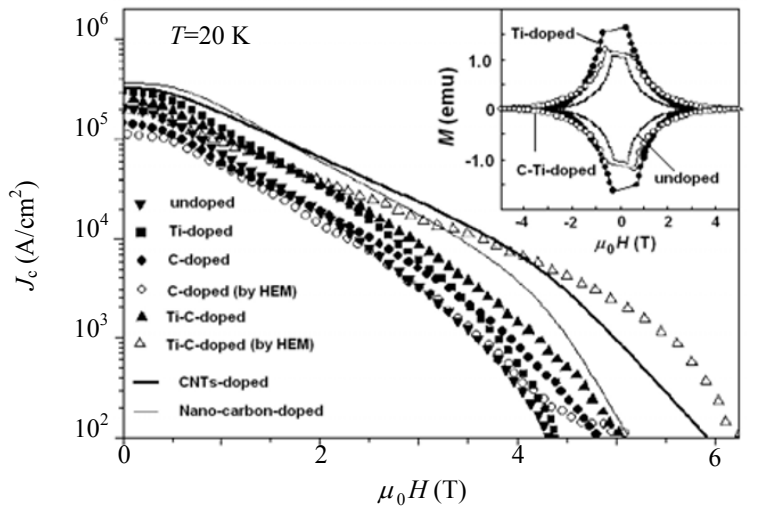

Fig. $3 J_{\mathrm{c}}(H)$ behavior at $\mathrm{T}=20 \mathrm{~K}$ for the undoped, Ti-doped, $\mathrm{C}$-doped, and C-Ti-doped samples. For the sake of comparison, the $J_{\mathrm{c}}$ results of nanocarbon-doped $\mathrm{MgB}_{2}$ [12] and CNTdoped $\mathrm{MgB}_{2}$ are plotted in the figure. Inset: Magnetization hysteresis loops at $\mathrm{T}=20 \mathrm{~K}$ for undoped, Ti-doped, and $\mathrm{C}$ - $\mathrm{Ti}$ doped samples.

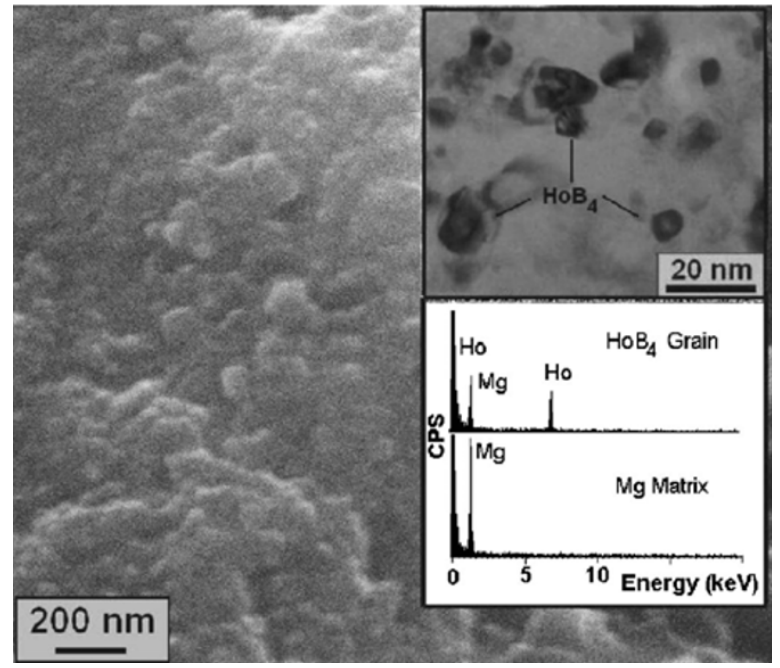

Fig. 4 TEM micrograph which shows the $\mathrm{HoB}_{4}$ nano-dots inserting in the $\mathrm{MgB}_{2}$ matrix, and EDX patterns for the nanoparticle shown in the TEM micrograph.

Fig. 5 shows the $J_{\mathrm{c}}(H)$ curves for $\mathrm{Mg}_{1-x}\left(\mathrm{Ho}_{2} \mathrm{O}_{3}\right)_{x} \mathrm{~B}_{2}$ samples at $5 \mathrm{~K}, 10 \mathrm{~K}$ and $20 \mathrm{~K}$. In the low-field region, the heavily-doped samples exhibit a $J_{\mathrm{c}}$ slightly lower than that of the undoped $\mathrm{MgB}_{2}$. However, at all temperatures studied in this work, the in-field critical current densities have been enhanced by $\mathrm{Ho}_{2} \mathrm{O}_{3}$ doping in high field region. The enhancement of $J_{\mathrm{c}}$ is getting more drastic with increasing doping level at $x \leqslant 3 \%$, but it begins to debase at $x=10 \%$, suggesting an optimal doping level for the $J_{\mathrm{c}}$ enhancement is between $3 \%$ and $10 \%$. In a field of $5 \mathrm{~T}$, the $3 \%$-doped $\mathrm{MgB}_{2}$ sample reaches a $J_{\mathrm{c}}$ of $1.0 \times 10^{3} \mathrm{~A} / \mathrm{cm}^{2}$ at $20 \mathrm{~K}, 2.0 \times 10^{4} \mathrm{~A} / \mathrm{cm}^{2}$ at $10 \mathrm{~K}$ and

\section{$1.2 \times 10^{5} \mathrm{~A} / \mathrm{cm}^{2}$ at $5 \mathrm{~K}$.}

Different from the doping effect of non-magnetic or weak magnetic impurities on $\mathrm{MgB}_{2}$, such as $\mathrm{Ti}, \mathrm{Zr}$, $\mathrm{Dy}_{2} \mathrm{O}_{3}$, and $\mathrm{Y}_{2} \mathrm{O}_{3}$ [3-7], which mainly improves the $J_{\mathrm{c}}$ in lower field region, $\mathrm{Ho}_{2} \mathrm{O}_{3}$ doping does not improve the low-field $J_{\mathrm{c}}$ significantly, but results in a significant improvement on $J_{\mathrm{c}}$ in higher field region. At $\mathrm{T}=5 \mathrm{~K}$, the $J_{\mathrm{c}}$ values of $\mathrm{Ti}$ - and $\mathrm{Dy}_{2} \mathrm{O}_{3}$-doped $\mathrm{MgB}_{2}$ are much higher than that of $\mathrm{Ho}_{2} \mathrm{O}_{3}$-doped sample when applied field is lower than $2 \mathrm{~T}$; however, these $J_{\mathrm{c}}$ values decrease rapidly with further increasing the applied field, reaching a $J_{\text {c }}$ value much lower than that of $\mathrm{Ho}_{2} \mathrm{O}_{3}$-doped sample in a field higher than $4 \mathrm{~T}$. For example, at $5 \mathrm{~K}$, both $10 \% \mathrm{Ti}$ and $3 \% \mathrm{Ho}_{2} \mathrm{O}_{3}$-doped $\mathrm{MgB}_{2}$ have a $J_{\mathrm{c}}$ around $2.0 \times 10^{5} \mathrm{~A} / \mathrm{cm}^{2}$ in a field of $4 \mathrm{~T}$, but the $J_{\mathrm{c}}$ for Ti-doped one drops to a value around $9.0 \times 10^{3} \mathrm{~A} / \mathrm{cm}^{2}$ at $6.5 \mathrm{~T}$ whereas $\mathrm{Ho}_{2} \mathrm{O}_{3}$-doped sample keeps a value higher than $7.0 \times 10^{4} \mathrm{~A} / \mathrm{cm}^{2}$, which is about 8 time higher. Further increasing the applied field to $9 \mathrm{~T}$, the $\mathrm{Ho}_{2} \mathrm{O}_{3}$-doped sample can still sustain a $J_{\mathrm{c}}$ as high as $3.0 \times 10^{4} \mathrm{~A} / \mathrm{cm}^{2}$. The improvement of $J_{\mathrm{c}}-\mathrm{H}$ behavior of $\mathrm{MgB}_{2}$ by $\mathrm{Ho}_{2} \mathrm{O}_{3}$ doping is also comparable with that achieved by nano- 
carbon-doped $\mathrm{MgB}_{2}$ in magnetic fields lower than $9 \mathrm{~T}$ [16] (see Fig. 5).

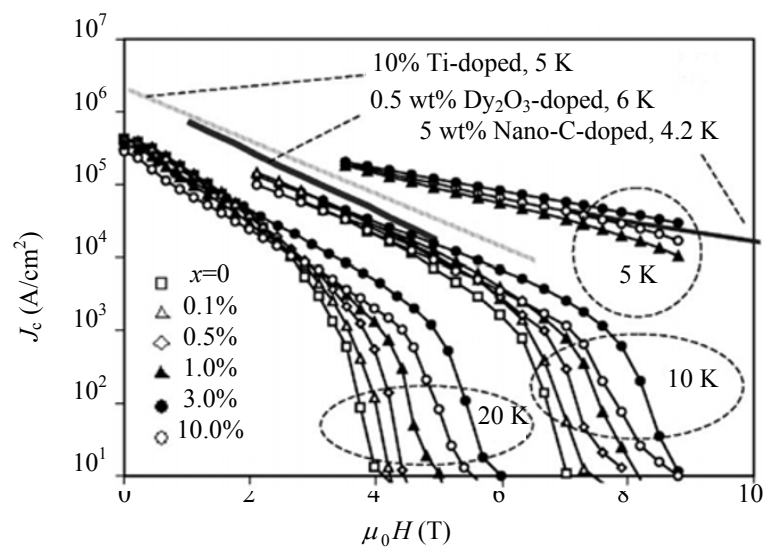

Fig. 5 Field dependence of magnetic $J_{\mathrm{c}}$ at 5,10 , and $20 \mathrm{~K}$ for $\mathrm{Mg}_{1-x}\left(\mathrm{Ho}_{2} \mathrm{O}_{3}\right)_{x} \mathrm{~B}_{2}$ samples with various doping levels [14$15]$.

\section{Conclusions}

From the comparison of recent research results on the nanostructural control of $\mathrm{MgB}_{2}$ by doping various nanoparticles including $\mathrm{Ti}$, nano-diamond, and $\mathrm{Ho}_{2} \mathrm{O}_{3}$, and their effects on flux pinning behavior of $\mathrm{MgB}_{2}$ some interesting conclusions can be drawn. (1) By nanodiamond doping, the diamond- $\mathrm{MgB}_{2}$ nano-composite superconductor forms, which consists of tightly-packed $\mathrm{MgB}_{2}$ nano-grains ( $\left.\sim 50-100 \mathrm{~nm}\right)$ with highly dispersed and uniformly distributed diamond nanoparticles $(\sim 10$ $20 \mathrm{~nm}$ ) inside the grains. (2) Carbon and Ti concurrently doping are largely cooperative in improving the performance of $\mathrm{MgB}_{2}$ in the high magnetic fields $(>3 \mathrm{~T})$ and at high temperature $(\sim 20 \mathrm{~K})$. (3) $\mathrm{In} \mathrm{Ho}_{2} \mathrm{O}_{3}$ doped samples, the magnetic $\mathrm{HoB}_{4}$ nanoparticles were attributed to be the source for the enhanced flux pinning effects. (4) Magnetic pinning centers show the most effective pinning behavior amongst those mentioned above and should be paid close attention in the practical applications.

\section{Acknowledgment}

This work was supported Australian Research Council (Nos. DP0559872 and DP0881739).

\section{References}

[1] J. Nagamatsu, N. Nakagawa, T. Muranaka, et al., Superconductivity at $39 \mathrm{~K}$ in magnesium diboride, Nature, 2001, 410: 63-64.
[2] A. Gurevich, Enhancement of the upper critical field by nonmagnetic impurities in dirty two-gap superconductors, Physical Review B, 2003, 67(18): 1-13.

[3] J. Wang, Y. Bugoslavsky, A. Berenov, et al., High critical current density and improved irreversibility field in bulk $\mathrm{MgB}_{2}$ made by a scaleable, nanoparticle addition route, Applied Physics Letters, 2002, 81(11): 2026-2028.

[4] Y. Zhao, D. X. Huang, Y. Feng, et al., Nanoparticle structure of $\mathrm{MgB}_{2}$ with ultrathin $\mathrm{TiB}_{2}$ grain boundaries, Applied Physics Letters, 2002, 80(9): 1640-1642.

[5] Y. Zhao, Y. Feng, C. H. Cheng, et al., High critical current density of $\mathrm{MgB}_{2}$ bulk superconductor doped with $\mathrm{Ti}$ and sintered at ambient pressure, Applied Physics Letters, 2001, 79(8): 1154-1156.

[6] S.K. Chen, M. Wei, J.L. MacManus-Driscoll, Strong pinning enhancement in $\mathrm{MgB}_{2}$ using very small $\mathrm{Dy}_{2} \mathrm{O}_{3}$ additions, Applied Physics Letters, 2006, 88(19): 192512-19512-3.

[7] Y. Feng, Y. Zhao, Y.P. Sun, et al., Improvement of critical current density in $\mathrm{MgB}_{2}$ superconductors by $\mathrm{Zr}$ doping at ambient pressure, Applied Physics Letters, 2001, 79(24): 3983-3985.

[8] C.H. Cheng, H. Zhang, Y. Zhao, et al., Doping effect of nano-diamond on superconductivity and flux pinning in $\mathrm{MgB}_{2}$, Superconductor Science and Technology, 2003, 16(10), 1182-1186.

[9] Y. Zhao, C.H. Cheng, X.F. Rui, et al., Improved irreversibility behavior and critical current density in MgB2-diamond nanocomposites, Applied Physics Letters, 2003, 83(14): 2916-2918.

[10] C.H. Cheng, Y. Yang, P. Munroe, et al., Comparison between nano-diamond and carbon nanotube doping effects on critical current density and flux pinning in $\mathrm{MgB}_{2}$, Superconductor Science and Technology, 2007, 20(3): 296-301.

[11] M. Xu, H. Kitazawa, Y. Takano, et al., Anisotropy of superconductivity from $\mathrm{MgB}_{2}$ single crystals, Applied Physics Letters, 2001, 79(17): 2779-2781.

[12] S. Soltanian, J. Horvat, X.L. Wang, et al., Effect of nano-carbon particle doping on the flux pinning properties of $\mathrm{MgB}_{2}$ superconductor, Physica C: Superconductivity, 2003, 390(3): 185.

[13] S.X. Dou, W.K. Yeoh, J. Horvat, et al., Effect of carbon nanotube doping on critical current density of $\mathrm{MgB} 2 \mathrm{su}-$ perconductor, Applied Physics Letters, 2003, 83(24): 4996-4998.

[14] C. Cheng, Y. Zhao, Significant improvement of flux pinning and irreversibility field of nano- $\mathrm{Ho}_{2} \mathrm{O}_{3}$ doped $\mathrm{MgB}_{2}$, Physica C: Superconductivity, 2007, 463-465: 220-224.

[15] C. Cheng, Y. Zhao, Enhancement of critical current density of $\mathrm{MgB}_{2}$ by doping $\mathrm{Ho}_{2} \mathrm{O}_{3}$, Applied Physics Letters, 2006, 89(25): 252501-252501-3.

[16] Y.W. Ma, X.P. Zhang, G. Nishijima, et al., Significantly enhanced critical current densities in $\mathrm{MgB}_{2}$ tapes made by a scaleable nanocarbon addition route, Applied Physics Letters, 2006, 88(7): , 072502-072502-2.

(Editor: Yao ZHOU) 